\title{
Editorial
}

\section{In the race for protection, ARF comes second}

\author{
$\mathrm{L} \mathrm{Golomb}^{*, 1}$ and M Oren ${ }^{1}$ \\ Cell Death and Differentiation (2013) 20, 1442-1443; doi:10.1038/cdd.2013.117
}

Many physiological processes require cell proliferation, starting with the rapid division of progenitor cells during embryogenesis, through the fast turnover of skin, intestinal and bone marrow-derived cells, and up to the robust cell proliferation during wound healing. In all those cases a cellular proliferation program needs to be executed, yet be terminated in a timely, precisely controlled manner. When the machinery in charge of regulating this process breaks down, cancer develops. Conversely, overreaction of the shutoff machinery might compromise tissue integrity, possibly spawning developmental defects, organ dysfunction and accelerated aging. Keeping the delicate balance between normal and excessive proliferation is the prime function of many tumor suppressors, hence their frequent loss in cancer. Proper preservation of cell and tissue homeostasis is safeguarded through an intricate, multilayered network of positive and negative feedback connections between many tumor suppressors and protooncogenes. Recent findings reveal a new thread in this fabric, involving an unexpected relationship between the DNA damage response (DDR) and the ARF tumor suppressor.

The CDKN2A (INK4a) locus might be described as a genetic marvel. The two proteins encoded by this locus differ by only a single exon, yet through translation of an alternative reading frame two completely different tumor suppressors are born. ${ }^{1}$ One is $\mathrm{p} 16^{\text {ink4a }}$, a cyclin-dependent kinase (CDK) inhibitor and a major component of the Rb tumor suppressor pathway. The other is the small, highly basic and largely unstructured p14ARF (p19ARF in mice). ARF is believed to exert most of its tumor suppressive capabilities via the p53 pathway. ${ }^{2}$ Through inhibiting the E3 ubiquitin ligase Mdm2, the major negative regulator of $\mathrm{p} 53$, ARF promotes accumulation and activation of p53, potentially resulting in apoptosis or senescence. Subsequently, ARF was also rediscovered as a p53-independent tumor suppressor, mainly through inhibition of ribosome biogenesis., ${ }^{3,4}$ Interestingly, ARF expression is attenuated by $\mathrm{p} 53$, underscoring a sophisticated regulatory crosstalk between the two tumor suppressors. ${ }^{2}$

Induction of functional ARF is commonly believed to be an immediate consequence of excessive mitogenic signaling, as occurs upon aberrant activation of many oncogenes. ${ }^{5,6}$ This view is now refined by Evangelou et al. ${ }^{7}$ in this issue of Cell Death and Differentiation. Specifically, these authors show that ARF induction is not an early outcome of oncogene activation. Rather, the immediate consequence of oncogene deregulation is activation of the DDR, instigated by the DNA replication stress elicited by excessive proliferative drive. ${ }^{8}$ When the authors examined a variety of mouse and human tumors, ARF protein was detected only in more advanced tumors. In contrast, DDR markers such as $\gamma \mathrm{H} 2 \mathrm{AX}$ and phospho-CHK2 were already evident in early, pre-neoplastic lesions. The notion that ARF is a late responder was reinforced by analysis of human bronchial epithelial cells and normal fibroblasts, transduced with various oncogenes. While single oncogenes efficiently triggered the DDR, only a combination of two different oncogenes could induce ARF accumulation (Figure 1). This implies the existence of a stress threshold that needs to be crossed in order to activate an ARF-driven antiproliferative program.

Existence of a stress threshold for ARF/p53-dependent antiproliferative responses is not a new concept. Several years ago Murphy et al. ${ }^{9}$ already reported that low levels of the c-Myc oncoprotein, while sufficient to transform cells, do not activate the ARF/p53 pathway; only when c-Myc is abundantly overexpressed does this pathway become activated, leading to apoptosis. Presumably, the ability of limited oncogenic signaling to 'go under the radar' of tumor suppressive checkpoints is meant to prevent undesirable triggering of these checkpoints during physiological responses, such as tissue renewal and wound healing. However, it also provides a loophole through which mild oncogene activation may go unpunished, at least during early stages of tumorigenesis.

How are such thresholds set? And is it just a coincidence that the threshold for DDR activation is lower than for ARF? Two recent studies reveal that the DDR actually actively maintains the ARF threshold high by promoting ARF protein degradation. ${ }^{10,11}$ The mastermind behind this scheme is the ATM kinase, a pivotal component of the DDR (Figure 1). When DNA damage is induced, ATM phosphorylates and activates the PP1 phosphatase, which then dephosphorylates the nucleolar protein NPM/B23. Phosphorylation of NPM (by the Nek2 kinase) is required in order to anchor ARF in the nucleolus, where it is stable and functional. When NPM is dephosphorylated by PP1, ARF disengages from the nucleolus and ends up in the nucleoplasm, where it falls prey to its E3 ubiquitin ligase ULF, resulting in ARF degradation. ${ }^{10}$ In this manner, the DDR keeps ARF protein low and prevents the triggering of the ARF/p53 axis. Indeed, depletion or inhibition of ATM elicit ARF accumulation (Figure 1, inset), with resultant activation of both p53-dependent and p53-independent antiproliferative programs such as inhibition of ribosome

${ }^{1}$ Department of Molecular Cell Biology, Weizmann Institute of Science, Rehovot, Israel

${ }^{*}$ Corresponding author: M Oren, Department of Molecular Cell Biology, Weizmann Institute of Science, POB 26, Rehovot 76100, Israel. Tel: + 9728 9342358; Fax: + 9728 9346004; E-mail: lior.golomb@weizmann.ac.il and moshe.oren@weizmann.ac.il 

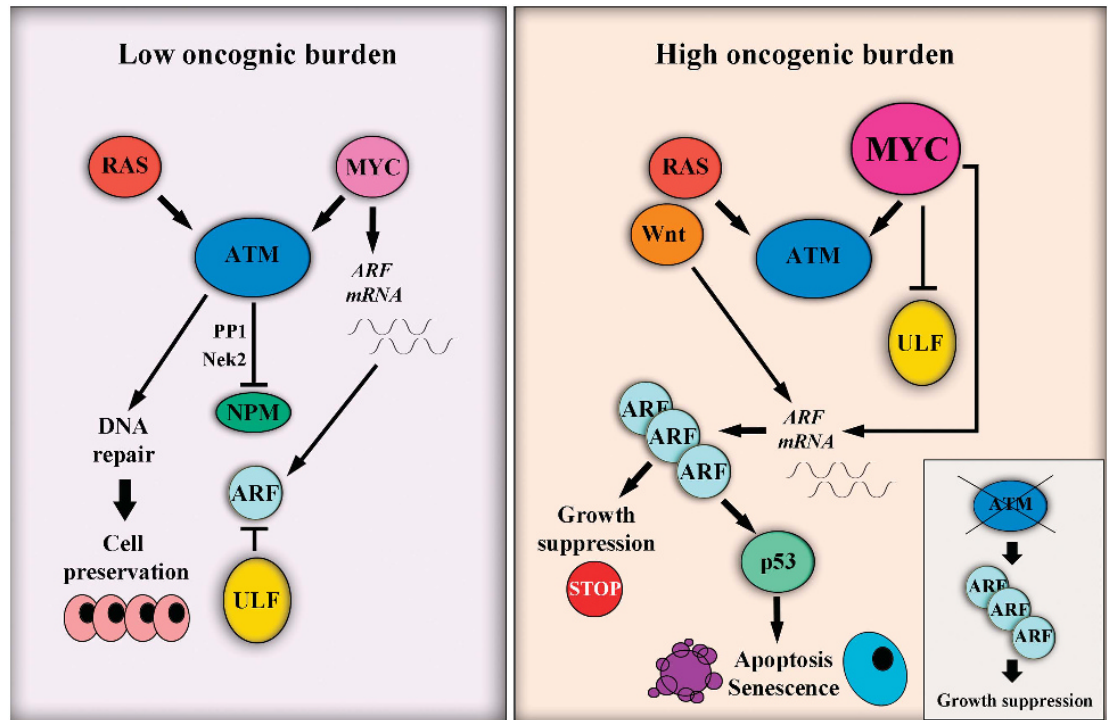

Figure 1 Schematic representation of ARF regulation by differential oncogenic burden. Left: low oncogenic burden, as exemplified by Ras activation alone, activates ATM and the DDR. ATM promotes ULF-mediated ARF degradation, preventing ARF accumulation. Mild c-Myc activation induces ARFmRNA, but ARF protein is not accumulated. Thus, the ARF activation threshold is not reached. Right: high oncogenic burden, as achieved by a combination of two oncogenes (exemplified by Ras and Wnt pathway activation) will override the inhibitory effect of the DDR and enable ARF accumulation. Likewise, excessive c-Myc will inhibit ULF and enable effective ARF accumulation. Thus, the ARF activation threshold is reached, eliciting ARF-driven, p53-dependent and p53-independent antiproliferative effects

biogenesis. ${ }^{10}$ The relevance of this mechanism is underscored by the inverse correlation between ARF and ATM levels in human lung tumors, where ATM is frequently mutated. ${ }^{10}$

So how does higher oncogenic stress nevertheless induce ARF, despite the persistence of DNA damage and DDR activation? At least for $\mathrm{C}-\mathrm{Myc}$, the answer appears rather simple: c-Myc binds directly to ULF, disrupting its interaction with ARF. Hence, when c-Myc reaches high enough levels, ARF remains protected from ULF even when the DDR drives it out of its safe nucleolar haven. ${ }^{11}$ The outcome is ARF accumulation, effectively underpinning the threshold described by Murphy et al..$^{9}$ (Figure 1).

Most certainly the overall picture is more complicated, varying among different oncogenes, cell types and animal species. For example, relatively low levels of c-Myc already induce transcription of $A R F$ mRNA, and the threshold for the checkpoint response is defined strictly by ARF protein stabilization. ${ }^{11}$ In contrast, in several scenarios involving the sequential activation of other oncogenes, including Ras, induction of $A R F$ mRNA is a critical component of the threshold. ${ }^{7}$ Regardless of the exact threshold mechanisms, these recent findings teach us that the cellular response to oncogenic stress is not controlled by a simple on/off switch. Rather, a sophisticated, dynamic program for mounting proportionate responses to progressive stress is in place. In this manner, cells that accumulate mild, spontaneous DNA damage during normal cell division are allowed to activate repair mechanisms while preserving their viability and proliferation potential. This also keeps at bay cells with mild oncogenic activation, where induction of the DDR is enough to arrest proliferation and prevent further neoplastic progression. Only when the oncogenic burden crosses a deleterious threshold and primary layers of protection fail, then does the secondary failsafe mechanism in the form of ARF activation get into action, with more severe consequences for the cell.

\section{Conflict of Interest}

The authors declare no conflict of interest.

1. Quelle DE et al. Cell 1995; 83: 993-1000.

2. Sherr CJ. Nat Rev Cancer 2006; 6: 663-673.

3. Sugimoto $\mathrm{M}$ et al. Mol Cell 2003; 11: 415-424.

4. Lessard F et al. Mol Cell 2010; 38: 539-550.

5. Zindy F et al. Genes Dev 1998; 12: 2424-2433.

6. Bates $S$ et al. Nature 1998; 395: 124-125.

7. Evangelou K et al. Cell Death Diff 2013; e-pub ahead of print 12 July 2013; PMID: 23852374.

8. Halazonetis TD, Gorgoulis VG, Bartek J. Science 2008; 319: 1352-1355.

9. Murphy DJ et al. Cancer Cell 2008; 14: 447-457.

10. Velimezi $\mathrm{G}$ et al. Nat Cell Biol 2013; 15: 967-977.

11. Chen D et al. Mol Cell 2013; 51: 46-56. 